STRUCTURAL BIOLOGY COMMUNICATIONS

ISSN 2053-230X
Received 13 October 2021

Accepted 28 November 2021

Edited by I. Tanaka, Hokkaido University, Japan

Keywords: SSGCID; structural genomics; Paraburkholderia xenovorans; oxidoreductases; education and training; detoxification; Seattle Structural Genomics Center for Infectious Disease.

PDB reference: putative short-chain reductase, 5 jc8

Supporting information: this article has supporting information at journals.iucr.org/f

\section{Crystal structure of a putative short-chain dehydrogenase/reductase from Paraburkholderia xenovorans}

\author{
Jaysón Davidson, ${ }^{a}$ Kyndall Nicholas, ${ }^{a}$ Jeremy Young, ${ }^{a}$ Deborah G. Conrady,, ,c \\ Stephen Mayclin,, ${ }^{b, c}$ Sandhya Subramanian, ${ }^{\text {c,d }}$ Bart L. Staker, ${ }^{c, d}$ Peter J. Myler,d and \\ Oluwatoyin A. Asojo ${ }^{\mathrm{a} *}$
}

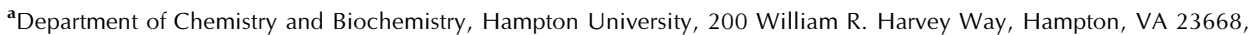
USA, ' ${ }^{\mathbf{U}}$ CB Pharma, Bedford, Massachusetts, USA, 'Seattle Structural Genomics Center for Infectious Disease (SSGCID), Seattle, Washington, USA, and ${ }^{\mathbf{d} C e n t e r}$ for Global Infectious Disease Research, Seattle Children's Research Institute, 307 Westlake Avenue North Suite 500, Seattle, WA 98109, USA. *Correspondence e-mail: oluwatoyin.asojo@hamptonu.edu

Paraburkholderia xenovorans degrades organic wastes, including polychlorinated biphenyls. The atomic structure of a putative dehydrogenase/reductase (SDR) from $P$. xenovorans ( $P x \mathrm{SDR}$ ) was determined in space group $P 2_{1}$ at a resolution of $1.45 \AA$. PxSDR shares less than $37 \%$ sequence identity with any known structure and assembles as a prototypical SDR tetramer. As expected, there is some conformational flexibility and difference in the substrate-binding cavity, which explains the substrate specificity. Uniquely, the cofactor-binding cavity of PxSDR is not well conserved and differs from those of other SDRs. PxSDR has an additional seven amino acids that form an additional unique loop within the cofactor-binding cavity. Further studies are required to determine how these differences affect the enzymatic functions of the SDR.

\section{Introduction}

Paraburkholderia xenovorans is a model bacterium used to study unique pathways, notably the ability of this genus of bacteria to degrade polychlorinated biphenyls and other organic waste products (Francova et al., 2004; Tehrani et al., 2014). The genome of $P$. xenovorans has been sequenced and is one of the largest bacterial genomes studied to date. $P$. xenovorans has diverse functions, including nitrogen fixation, the catabolysis of aromatic compounds and the degradation of various organic wastes (Francova et al., 2004; Tehrani et al., 2014). However, the underlying mechanisms of many of these processes are poorly understood. Additionally, unlike some other Paraburkholderia species, P. xenovorans is not pathogenic to humans. The Seattle Structural Genomics Center for Infectious Disease (SSGCID) selected targets from $P$. xenovorans for high-throughput structural studies to increase the breadth of drug-target structures to aid drug development against human pathogenic Burkholderia species such as $B$. mallei and B. pseudomallei. Here, we present the structure of one of these target proteins, a short-chain dehydrogenase/reductase (SDR) which shares less than $37 \%$ sequence identity with any published structure. P. xenovorans SDR ( $P x$ SDR) is predicted to be a type II fatty-acid synthetase and $\mathrm{NAD}(\mathrm{P})(\mathrm{H})$-dependent oxidoreductase involved in the metabolism of diverse molecules, including lipids, amino acids, carbohydrates, cofactors, hormones and xenobiotics, or other compounds. The structure reported here may offer insights into the metabolism of small molecules by $P$. xenovorans. 
Table 1

Macromolecule-production information.

\begin{tabular}{|c|c|}
\hline Source organism & Paraburkholderia xenovorans (strain LB400) \\
\hline DNA source & $\begin{array}{l}\text { Genomic DNA, provided by Dr Mary } \\
\text { Lidstrom (University of Washington, } \\
\text { USA) }\end{array}$ \\
\hline Forward primer & $\begin{array}{l}\text { 5'-CTCACCACCACCACCACCATATGAGTTCA } \\
\text { GCAGGAAGATTGCAG-3' }\end{array}$ \\
\hline Reverse primer & $\begin{array}{l}5^{\prime} \text {-АTCCTATCTTACTCACTTAATCGCAGCGG } \\
\text { GCGCTCATCC-3' }\end{array}$ \\
\hline Expression vector & 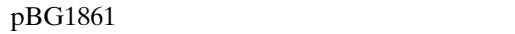 \\
\hline Expression host & Escherichia coli BL21(DE3)R3 Rosetta cells \\
\hline $\begin{array}{l}\text { Complete amino-acid sequence } \\
\text { of the construct produced }\end{array}$ & $\begin{array}{l}\text { MAHHHHHHMSSAGRLQGKVALVTGAGCIGPG } \\
\text { WGNGRAIAVRFAEEGAHVIAVDRDLASMD } \\
\text { ATLELVRAAGGSVTPCLCDVTDSASVERL } \\
\text { VADSVARCGRVDILVNNVGAPSPGGPVAL } \\
\text { DEAQWAMQLELNLTTAFLMCKYVLPVMEQ } \\
\text { QGGGAIVNIASTSGIRWTGAAQVGYAAAK } \\
\text { AGMIQMGRVVAVEYAAKNVRVNSVVPGLL } \\
\text { HTPMVDTKIAHNQAGGDVELLLRKRQARI } \\
\text { PMPFMGDGRDTANAALFLASDEARFVTGT } \\
\text { EIVVDGGMSARCD }\end{array}$ \\
\hline
\end{tabular}

\section{Materials and methods}

\subsection{Macromolecule production}

Cloning, expression and purification were conducted as part of the Seattle Structural Genomics Center for Infectious Disease (SSGCID; Myler et al., 2009; Stacy et al., 2011) following standard protocols described previously (Bryan et al., 2011; Choi et al., 2011; Serbzhinskiy et al., 2015). The fulllength putative short-chain reductase (PxSDR, UniProt Q13GE3) was PCR-amplified from genomic DNA using the primers shown in Table 1. The resultant amplicon was cloned into the ligation-independent cloning (LIC; Aslanidis \& de Jong, 1990) expression vector pBG1861 encoding a noncleavable $6 \times$ His fusion tag (MAHHHHHHM-ORF). Plasmid DNA was transformed into chemically competent Escherichia coli BL21(DE3)R3 Rosetta cells. The plasmid containing HisPxSDR was expression-tested and 21 of culture was grown using auto-induction medium (Studier, 2005) in a LEX Bioreactor (Epiphyte Three Inc.). The expression clone for PxSDR, BuxeA.00010.c.B1.GE39410, is available at https:// www.ssgcid.org/available-materials/expression-clones/.

His- $P x$ SDR was purified in a two-step protocol consisting of an immobilized metal-affinity chromatography (IMAC) step and size-exclusion chromatography (SEC). All chromatography runs were performed on an ÄKTApurifier 10 (GE) using automated IMAC and SEC programs according to previously described procedures (Bryan et al., 2011). Thawed bacterial pellets were lysed by sonication in $200 \mathrm{ml}$ buffer consisting of $25 \mathrm{~m} M$ HEPES $\mathrm{pH} 7.0,500 \mathrm{~m} M \mathrm{NaCl}, 5 \%$ glycerol, $0.5 \%$ CHAPS, $30 \mathrm{~m} M$ imidazole, $10 \mathrm{~m} M \mathrm{MgCl}_{2}$, $1 \mathrm{~m} M$ TCEP, $250 \mu \mathrm{g} \mathrm{ml}^{-1}$ AEBSF, $0.025 \%$ azide. After sonication, the crude lysate was clarified with $20 \mu \mathrm{l}\left(25\right.$ units $\left.\mu^{-1}\right)$ benzonase and incubated while mixing at room temperature for $45 \mathrm{~min}$. The lysate was then clarified by centrifugation at

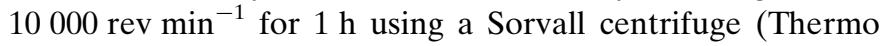
Scientific). The clarified supernatant was then passed over an Ni-NTA HisTrap FF $5 \mathrm{ml}$ column (GE Healthcare) which was pre-equilibrated with loading buffer composed of $25 \mathrm{mM}$ HEPES pH 7.0, $500 \mathrm{~m} \mathrm{M} \mathrm{NaCl,} \mathrm{5 \%} \mathrm{glycerol,} 30 \mathrm{~m} M$ imidazole,
Table 2

Crystallization.

\begin{tabular}{|c|c|}
\hline Method & Vapor diffusion, sitting drop \\
\hline Plate type & 96-well, Compact 300, Rigaku \\
\hline Temperature (K) & 287 \\
\hline Protein concentration & $\begin{array}{l}\text { PxSDR (BuxeA.00010.c.B1.PS02595) at } \\
\quad 22.5 \mathrm{mg} \mathrm{ml}^{-1}\end{array}$ \\
\hline $\begin{array}{l}\text { Buffer composition of protein } \\
\text { solution }\end{array}$ & $\begin{array}{l}25 \mathrm{~m} M \text { HEPES pH 7.0, } 500 \mathrm{~m} M \mathrm{NaCl} \\
5 \% \text { glycerol, } 2 \mathrm{~m} M \text { DTT, } 0.025 \% \text { azide }\end{array}$ \\
\hline $\begin{array}{l}\text { Composition of reservoir } \\
\text { solution }\end{array}$ & $\begin{array}{l}\text { Rigaku Reagents JCSG+ screen H11: } 0.2 M \\
\text { magnesium chloride, } 0.1 M \text { bis-Tris pH 5.5, } \\
25 \% \text { PEG } 3350\end{array}$ \\
\hline & $0.4 \mu \mathrm{l}$ protein: $0.4 \mu \mathrm{l}$ reservoir $(1: 1)$ \\
\hline Volume of reservoir $(\mu \mathrm{l})$ & 80 \\
\hline
\end{tabular}

$1 \mathrm{~m} M$ TCEP, $0.025 \%$ sodium azide. The column was washed with 20 column volumes $(\mathrm{CV})$ of loading buffer and was eluted with loading buffer plus $250 \mathrm{~m} M$ imidazole in a linear gradient over $7 \mathrm{CV}$. Peak fractions, as determined by UV absorption at $280 \mathrm{~nm}$, were pooled and concentrated to $5 \mathrm{ml}$. A SEC column (Superdex 75, GE) was equilibrated with running buffer composed of $25 \mathrm{~m} M$ HEPES pH 7.0, $500 \mathrm{~m} M \mathrm{NaCl}, 5 \%$ glycerol, $2 \mathrm{~m} M$ DTT, $0.025 \%$ azide. The peak fractions were collected and analyzed for the protein of interest using SDSPAGE. The SEC peak fractions eluted as a single large peak at a molecular mass of $\sim 76 \mathrm{kDa}$, suggesting a dimeric enzyme. The peak fractions were pooled and concentrated to $45 \mathrm{mg} \mathrm{ml}^{-1}$ using an Amicon purification system (Millipore). Aliquots of $200 \mu \mathrm{l}$ were flash-frozen in liquid nitrogen and stored at $-193 \mathrm{~K}$ until use for crystallization.

\subsection{Crystallization}

PxSDR was crystallized by the sitting-drop vapor-diffusion method using the JCSG+ commercial crystallization screen (Rigaku Reagents). Crystals were obtained by mixing $0.4 \mu \mathrm{l}$ protein solution at $22.5 \mathrm{mg} \mathrm{ml}^{-1}$ with $0.4 \mu \mathrm{l}$ precipitant (Rigaku Reagents JCSG+ screen condition H11: $0.2 M$ magnesium chloride, $0.1 M$ bis-Tris pH 5.5, 25\% PEG 3350), equilibrating against a reservoir consisting of $80 \mu \mathrm{l}$ precipitant and incubating at $287 \mathrm{~K}$ (Table 2). A single crystal was transferred into a cryoprotectant (reservoir solution supplemented with $20 \%$ ethylene glycol) and vitrified by plunging into liquid nitrogen before data collection.

\subsection{Data collection and processing}

X-ray diffraction data were collected on LS-CAT beamline 21-ID-F at the Advanced Photon Source. Data were integrated using XDS and reduced with XSCALE (Kabash, 2010). Additional data-collection information is provided in Table 3. The raw images and detailed data-collection information are available for download (https://proteindiffraction.org/project/ $5 \mathrm{jc} 8 /)$.

\subsection{Structure solution and refinement}

The structure of $P x \mathrm{SDR}$ was solved by molecular replacement with MOLREP (Vagin \& Teplyakov, 2010; Lebedev et al., 2008) using PDB entry 4ni5 (36\% sequence identity), an unpublished structure from the SSGCID, as a search model. 
Table 3

Data collection and processing.

Values in parentheses are for the outer shell.

\begin{tabular}{ll}
\hline Diffraction source & Beamline 21-ID-F, APS \\
Wavelength $(\AA)$ & 0.97872 \\
Temperature $(\mathrm{K})$ & 100 \\
Detector & RayoniX MX-225 CCD \\
Crystal-to-detector distance $(\mathrm{mm})$ & 140 \\
Rotation range per image $\left(^{\circ}\right)$ & 1 \\
Total rotation range $\left(^{\circ}\right)$ & 240 \\
Exposure time per image $(\mathrm{s})$ & 1 \\
Space group & $P 2_{1}$ \\
$a, b, c(\AA)$ & $71.18,81.08,86.37$ \\
$\alpha, \beta, \gamma\left({ }^{\circ}\right)$ & $90,94.53,90$ \\
Mosaicity $\left(^{\circ}\right)$ & 0.145 \\
Resolution range $(\AA)$ & $50-1.45(1.49-1.45)$ \\
Total No. of reflections & $855886(53861)$ \\
No. of unique reflections & $165687(10796)$ \\
Completeness $(\%)$ & $95.8(84.8)$ \\
Multiplicity & $5.2(5.0)$ \\
$\langle I / \sigma(I)\rangle$ & $25.10(4.45)$ \\
$R_{\text {r.i.m. }}{ }^{\dagger}$ & $0.042(0.392)$ \\
Overall $B$ factor from Wilson plot $\left(\AA^{2}\right)$ & 12.76
\end{tabular}

$\dagger$ Estimated $R_{\text {r.i.m. }}=R_{\text {merge }}[N /(N-1)]^{1 / 2}$, where $N$ is the data multiplicity.

Table 4

Structure solution and refinement.

Values in parentheses are for the outer shell

\begin{tabular}{ll}
\hline Resolution range $(\AA)$ & $50-1.45(1.48-1.45)$ \\
Completeness (\%) & 95.8 \\
$\sigma$ Cutoff & $F>1.34 \sigma(F)$ \\
No. of reflections, working set & $165652(9476)$ \\
No. of reflections, test set & $2032(112)$ \\
Final $R_{\text {cryst }}$ & $0.141(0.194)$ \\
Final $R_{\text {free }}$ & $0.158(0.202)$ \\
No. of non-H atoms & \\
$\quad$ Protein & 7374 \\
$\quad$ Ion & 4 \\
$\quad$ Ligand & 66 \\
$\quad$ Solvent & 1068 \\
$\quad$ Total & 8512 \\
R.m.s. deviations & \\
$\quad$ Bonds $(\AA)$ & 0.007 \\
$\quad$ Angles ( $)$ & 0.878 \\
Average $B$ factors $\left(\AA^{2}\right)$ & \\
$\quad$ Protein & 17.5 \\
$\quad$ Ion & 17.7 \\
$\quad$ Ligand & 33.7 \\
$\quad$ Water & 31.7 \\
Ramachandran plot & \\
$\quad$ Most favored $(\%)$ & 96 \\
$\quad$ Allowed $(\%)$ & 4 \\
\hline
\end{tabular}

The structure was refined in Phenix (Liebschner et al., 2010) with manual model building in Coot (Emsley \& Cowtan, 2004; Emsley et al., 2010). The quality of the model was assessed using MolProbity (Headd et al., 2009) and structure-refinement statistics are provided in Table 4. The structure was deposited in the Protein Data Bank as entry 5jc8. All structure figures were made using PyMOL (DeLano, 2002).

\section{Results and discussion}

The reported apo structure of $P x \mathrm{SDR}$ was determined in the monoclinic space group $P 2_{1}$ as a prototypical SDR tetramer
(Fig. 1). Each monomer has the prototypical NADPH Rossmann topology as observed in the architecture of PFAM domain PF00106 or the short-chain dehydrogenases. Specifically, PxSDR has the enoyl-(acyl carrier protein) reductase or 3-oxoacyl-ACP reductase domain architecture otherwise referred to as adh_short_C2. The most similar structures to PxSDR were identified by PDBeFold (http://www.ebi.ac.uk/ msd-srv/ssm) analysis using the default threshold cutoffs of $70 \%$ for the percentage of secondary structure of the target chain identified in the query protein and of the secondary structure of the query chain (Krissinel \& Henrick, 2004). The closest structure is that of the ketone reductase ChKRED20 from the genome of Chryseobacterium ( $\mathrm{Li}$ et al., 2019). This enzyme shares a sequence identity of $<36 \%$, with an r.m.s.d. of $1.24 \AA$ for $89 \%$ of the matched sequence identity. Wild-type ChKRED20 is an NADH-dependent ketoreductase that reduces over $100 \mathrm{~g} \mathrm{l}^{-1}$ ketones for some pharmaceutically relevant substrates and can use 2-propanol as the ultimate reducing agent. All of the closest structures have less than $36 \%$ sequence similarity to PxSDR (Fig. 2). These structures are PDB entry 1iy8, the crystal structure of levodione reductase from Leifsonia aquatica (Sogabe et al., 2003), PDB entry $3 \mathrm{ftp}$, a 3-ketoacyl-(acyl-carrier-protein) reductase from Burkholderia pseudomallei (Baugh et al., 2013), PDB entry 6t6n, Klebsiella pneumoniae FabG2(NADH-dependent) in complex with NADH (Vella et al., 2021), and PDB entry 6ixm, ketone reductase ChKRED20 from the genome of Chryseobacterium (Li et al., 2019). Interestingly, while all of the other structures have well conserved cofactor-binding domains, an extended loop connecting the first strand in the N-terminus to

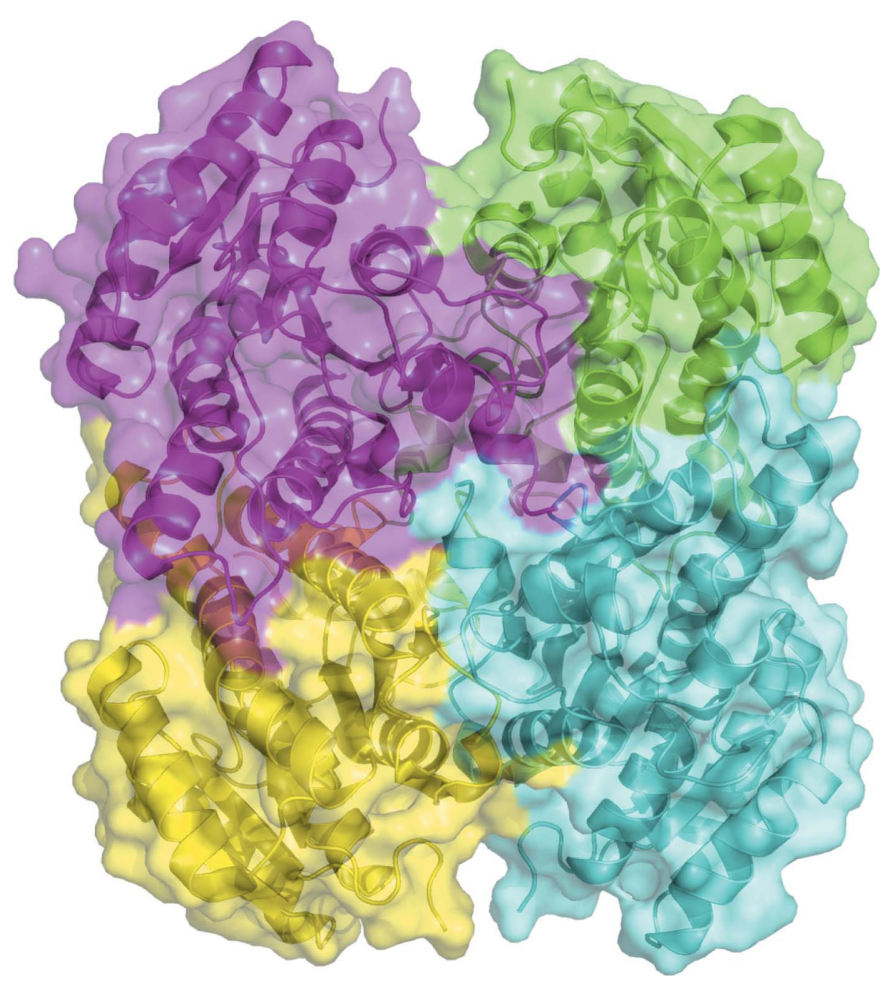

Figure 1

$P x \mathrm{SDR}$ is a prototypyical SDR tetramer. 


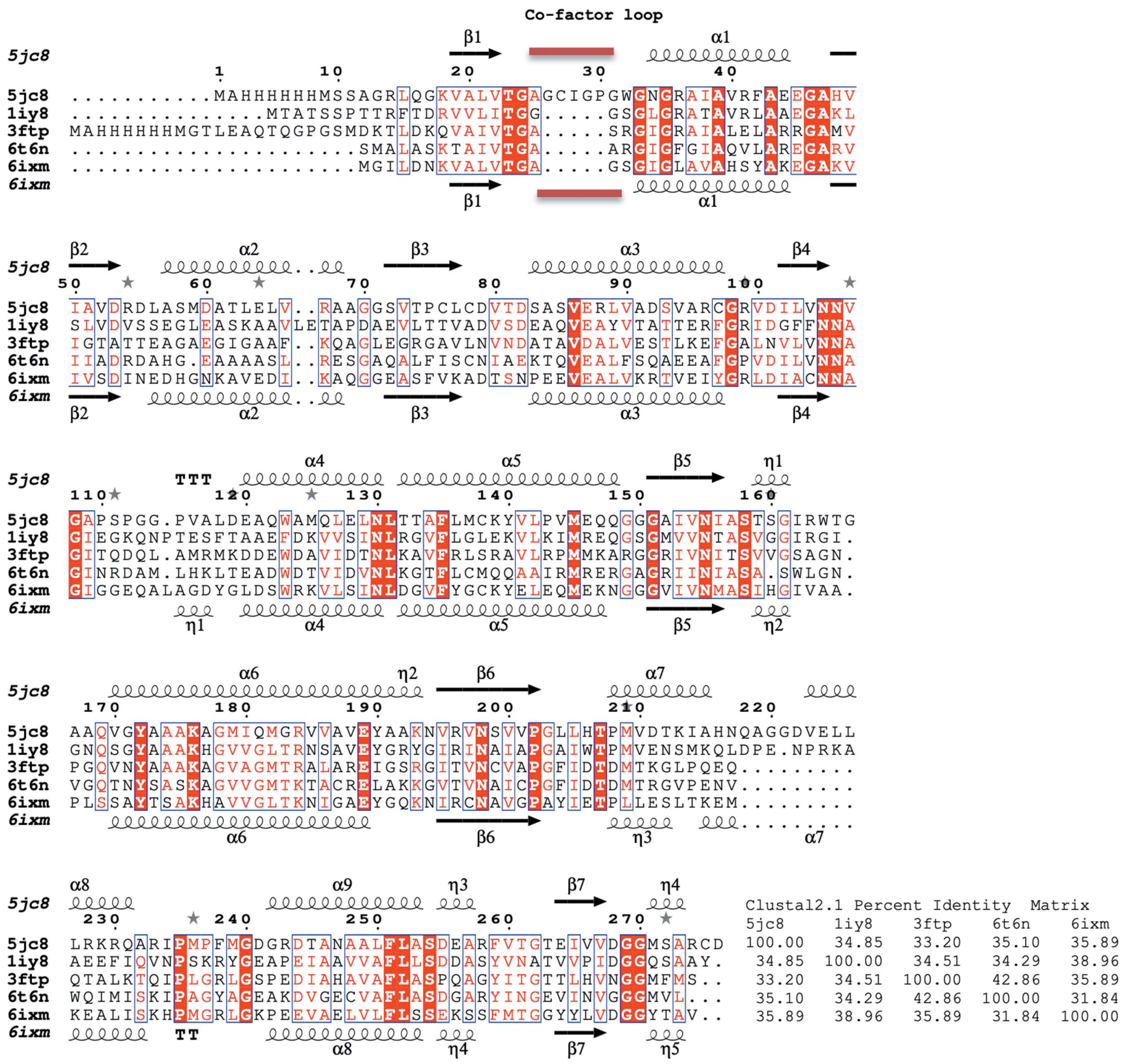

Figure 2

Structural and primary-sequence alignment of PxSDR with the closest structures identified by PDBeFold. Also shown is the percent identity matrix generated with Clustal2.1. The structures are PDB entry 5jc8 (the apo structure of PxSDR), PDB entry 1iy8 (the crystal structure of levodione reductase from Leifsonia aquatica), PDB entry 3ftp [3-ketoacyl-(acyl-carrier-protein) reductase from Burkholderia pseudomallei], PDB entry 6t6n [Klebsiella pneumoniae FabG2(NADH-dependent) in complex with NADH] and PDB entry 6ixm (ketone reductase ChKRED20 from the genome of Chryseobacterium). The secondary-structure elements shown are $\alpha$-helices $(\alpha), 3_{10}$-helices $(\eta), \beta$-strands $(\beta)$ and $\beta$-turns (TT). Identical residues are shown in white on a red background and conserved residues are shown in red. This figure was generated using ESPript (Gouet et al., 1999, 2003).

the first helix creates a larger cofactor-binding cavity in PxSDR (Figs. 2 and 3). This additional structural difference between PxSDR and the other structures in the cofactorbinding domain is unique. This is an unexpected difference between $P x \mathrm{SDR}$ and the other proteins beyond the expected flexibility in proximity to the substrate-binding cavity. While the flexibility in the substrate-binding cavity explains the specificity of each protein, it is unknown why $P x \mathrm{SDR}$ has this unique insertion in the cofactor loop (Figs. 2 and 3).

\section{Conclusions}

While having a prototypical SDR topology, the apo structure of PxSDR reveals conformational flexibility in both the 


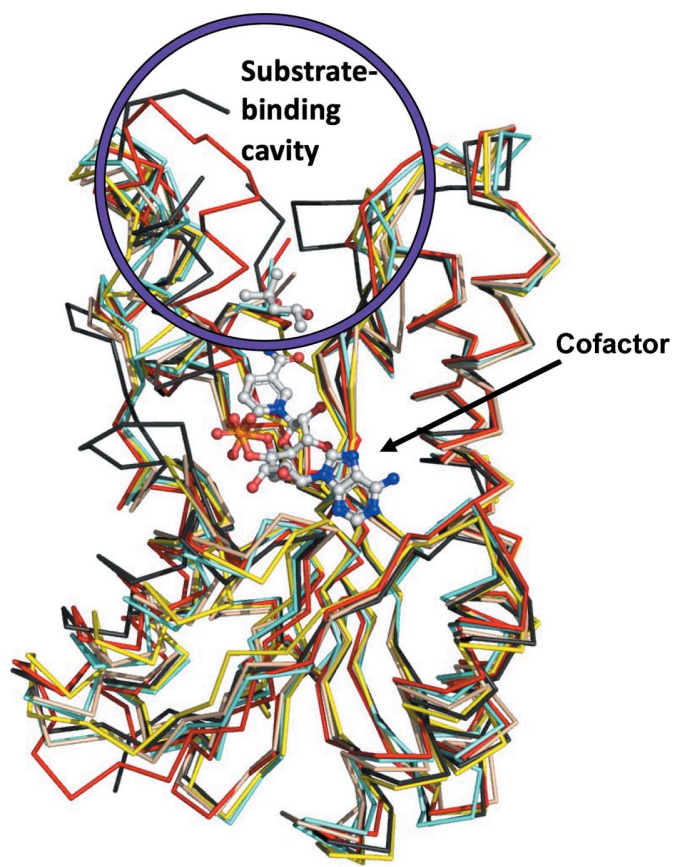

(a)

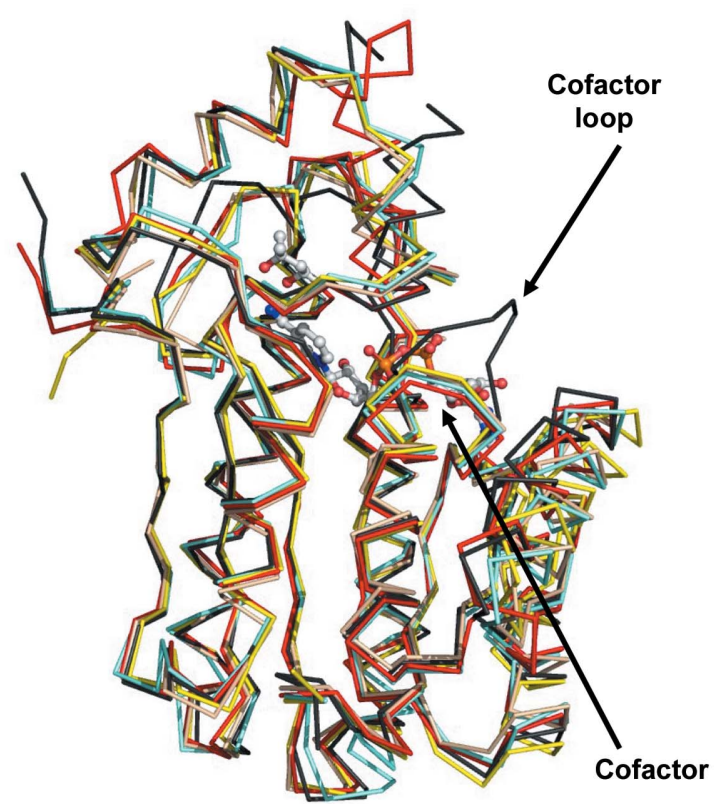

(b)

Figure 3

PxSDR superposed on its closest structural orthologues reveals differences in the substrate- and cofactor-binding cavities. (a) The substrate-binding cavities of the proteins, indicated in the blue circle, have differences that are indicative of different substrate specificities. (b) $P x$ SDR in black has a unique loop insertion in the cofactor-binding cavity, while the other proteins have a well conserved cofactor-binding cavity and loops. The superposed structures are PDB entry 5jc8 (apo structure of PxSDR, black), PDB entry 1iy8 (crystal structure of levodione reductase from Leifsonia aquatica, red), PDB entry 3ftp [3-ketoacyl-(acyl-carrier-protein) reductase from Burkholderia pseudomallei, yellow], PDB entry 6t6n [Klebsiella pneumoniae FabG2(NADH-dependent) in complex with NADH, wheat] and PDB entry 6ixm (ketone reductase ChKRED20 from the genome of Chryseobacterium, aquamarine). The cofactor, NADH and substrate, (4R)-2-methylpentane-2,4-diol, are from PDB entry 1iy8. Structures were superposed with $P y M O L$.

cofactor- and substrate-binding cavities that needs to be further investigated in order to determine the roles of this enzyme in the degradation of organic wastes by $P$. xenovorans.

\section{Acknowledgements}

We thank the SSGCID cloning and protein-production groups at the Center for Infectious Disease Research and the University of Washington. This research used resources of the Advanced Photon Source, a US Department of Energy (DOE) Office of Science User Facility operated for the DOE Office of Science by Argonne National Laboratory under Contract No. DE-AC02-06CH11357. Use of the LS-CAT Sector 21 was supported by the Michigan Economic Development Corporation and the Michigan Technology TriCorridor (Grant 085P1000817).

\section{Funding information}

This work was supported by the National Institutes of Health/ National Institute of Allergy and Infectious Diseases (contract Nos. HHSN272201700059C, HHSN272201200025C and HHSN272200700057C to PJM). JY was part of a pilot Hampton University Chemistry Education and Mentorship Course Based Undergraduate Research project (HU-ChEM CURES) funded by the NIGMS (1U01GM138433-01 to OAA).

\section{References}

Aslanidis, C. \& de Jong, P. J. (1990). Nucleic Acids Res. 18, 6069-6074.

Baugh, L., Gallagher, L. A., Patrapuvich, R., Clifton, M. C., Gardberg, A. S., Edwards, T. E., Armour, B., Begley, D. W., Dieterich, S. H., Dranow, D. M., Abendroth, J., Fairman, J. W., Fox, D., Staker, B. L., Phan, I., Gillespie, A., Choi, R., Nakazawa-Hewitt, S., Nguyen, M. T., Napuli, A., Barrett, L., Buchko, G. W., Stacy, R., Myler, P. J., Stewart, L. J., Manoil, C. \& Van Voorhis, W. C. (2013). PLoS One, 8, e53851.

Bryan, C. M., Bhandari, J., Napuli, A. J., Leibly, D. J., Choi, R., Kelley, A., Van Voorhis, W. C., Edwards, T. E. \& Stewart, L. J. (2011). Acta Cryst. F67, 1010-1014.

Choi, R., Kelley, A., Leibly, D., Nakazawa Hewitt, S., Napuli, A. \& Van Voorhis, W. (2011). Acta Cryst. F67, 998-1005.

DeLano, W. L. (2002). http://www.pymol.org

Emsley, P. \& Cowtan, K. (2004). Acta Cryst. D60, 2126-2132.

Emsley, P., Lohkamp, B., Scott, W. G. \& Cowtan, K. (2010). Acta Cryst. D66, 486-501.

Francova, K., Macková, M., Macek, T. \& Sylvestre, M. (2004). Environ. Pollut. 127, 41-48.

Gouet, P., Courcelle, E., Stuart, D. I. \& Métoz, F. (1999). Bioinformatics, 15, 305-308.

Gouet, P., Robert, X. \& Courcelle, E. (2003). Nucleic Acids Res. 31, 3320-3323.

Headd, J. J., Immormino, R. M., Keedy, D. A., Emsley, P., Richardson, D. C. \& Richardson, J. S. (2009). J. Struct. Funct. Genomics, 10, 8393.

Kabsch, W. (2010). Acta Cryst. D66, 125-132.

Krissinel, E. \& Henrick, K. (2004). Acta Cryst. D60, 2256-2268.

Lebedev, A. A., Vagin, A. A. \& Murshudov, G. N. (2008). Acta Cryst. D64, 33-39. 
Li, T. B., Zhao, F. J., Liu, Z., Jin, Y., Liu, Y., Pei, X. Q., Zhang, Z. G., Wang, G. \& Wu, Z. L. (2019). Enzyme Microb. Technol. 125, 29-36. Liebschner, D., Afonine, P. V., Baker, M. L., Bunkóczi, G., Chen, V. B., Croll, T. I., Hintze, B., Hung, L.-W., Jain, S., McCoy, A. J., Moriarty, N. W., Oeffner, R. D., Poon, B. K., Prisant, M. G., Read, R. J., Richardson, J. S., Richardson, D. C., Sammito, M. D., Sobolev, O. V., Stockwell, D. H., Terwilliger, T. C., Urzhumtsev, A. G., Videau, L. L., Williams, C. J. \& Adams, P. D. (2019). Acta Cryst. D75, 861-877.

Myler, P. J., Stacy, R., Stewart, L., Staker, B. L., Van Voorhis, W. C., Varani, G. \& Buchko, G. W. (2009). Infect. Disord. Drug Targets, 9 , 493-506.

Serbzhinskiy, D. A., Clifton, M. C., Sankaran, B., Staker, B. L., Edwards, T. E. \& Myler, P. J. (2015). Acta Cryst. F71, 594-599.
Sogabe, S., Yoshizumi, A., Fukami, T. A., Shiratori, Y., Shimizu, S., Takagi, H., Nakamori, S. \& Wada, M. (2003). J. Biol. Chem. 278, 19387-19395.

Stacy, R., Begley, D. W., Phan, I., Staker, B. L., Van Voorhis, W. C., Varani, G., Buchko, G. W., Stewart, L. J. \& Myler, P. J. (2011). Acta Cryst. F67, 979-984.

Studier, F. W. (2005). Protein Expr. Purif. 41, 207-234.

Tehrani, R., Lyv, M. M. \& Van Aken, B. (2014). Environ. Sci. Pollut. Res. 21, 6346-6353.

Vagin, A. \& Teplyakov, A. (2010). Acta Cryst. D66, 22-25.

Vella, P., Rudraraju, R. S., Lundbäck, T., Axelsson, H., Almqvist, H., Vallin, M., Schneider, G. \& Schnell, R. (2021). Bioorg. Med. Chem. 30, 115898 . 